\title{
Peritumoral Hyperintensity at Hepatobiliary Phase Gadoxetic Acid-enhanced MRI in Hepatic Neuroendocrine Tumors
}

\author{
NOBUHIRO FUJITA ${ }^{1,2}$, ATSUHIKO HANDA ${ }^{2}$ and JINHA M. PARK ${ }^{2}$ \\ ${ }^{1}$ Department of Clinical Radiology, Graduate School of Medical Sciences, Kyushu University, Fukuoka, Japan; \\ ${ }^{2}$ Department of Radiology, University of Iowa Hospitals and Clinics, Iowa City, IA, U.S.A.
}

\begin{abstract}
Background/Aim: To identify the imaging and clinical features of hepatic neuroendocrine tumors (NETs) associated with peritumoral hyperintensity in the hepatobiliary phase of gadoxetic acid-enhanced magnetic resonance (MR) imaging. Patients and Methods: Fifty-seven patients with hepatic NETs were enrolled. Based on the degree of peritumoral hyperintensity, patients were divided into three groups: group 0 (no peritumoral hyperintensity), group 1 (lower peritumoral hyperintensity), and group 2 (higher peritumoral hyperintensity). The imaging and clinical findings were compared among the three groups. Results: Apparent diffusion coefficient (ADC) values of group 2 were significantly lower than those of group 0 and group 1. Atypical (cholangiocarcinoma-like) enhancement pattern in the arterial phase was significantly more frequently observed in group 2 as compared to that in group 0 and group 1. Group 2 patients showed significantly poorer progression-free survival than group 0 patients. Conclusion: Hepatic NETs with greater peritumoral hyperintensity exhibit greater malignant potential.
\end{abstract}

Neuroendocrine tumors (NETs) originate in the gastrointestinal tract, pancreas, bronchopulmonary tree, and thyroid; these tumors exhibit a wide range of malignant potential $(1,2)$. The liver is the most common site of metastasis in patients with NETS; liver metastases are observed in approximately $80 \%$ of patients with metastases (3). Liver metastasis is one of the most important prognostic factors with respect to survival of patients with NETs (4). Therefore, once a diagnosis of NET is established, appropriate investigations such as computed tomography (CT) or magnetic

This article is freely accessible online.

Correspondence to: Nobuhiro Fujita, MD, Ph.D., Department of Clinical Radiology, Graduate School of Medical Sciences, Kyushu University, 3-1-1 Maidashi, Higashi-ku, Fukuoka, 812-8582, Japan. Tel: +81926425695, e-mail: fujita.nobuhiro.642@m.kyushu-u.ac.jp

Key Words: Neuroendocrine tumor, liver, gadoxetic acid, MRI. resonance (MR) imaging are needed. Gadoxetic acid is a hepatobiliary-specific contrast medium for MR imaging, which is taken up by functioning hepatocytes $(5,6)$. Tumors such as NETs that lack functioning hepatocytes usually show hypointensity compared to the background liver in the hepatobiliary phase (HBP) (5). Studies have shown a higher sensitivity of gadoxetic acid-enhanced MR imaging in the detection of hepatic metastases $(7,8)$.

In clinical practice, we sometimes encounter hepatic NETs that show hyperintense rim surrounding the tumor (peritumoral hyperintensity) in the HBP of gadoxetic acidenhanced MR imaging. The mechanism of peri-tumoral hyperintensity is not clear. According to a recent study, peritumoral hyperintensity in hepatocellular carcinoma (HCC) suggests histological peri-tumoral hyperplasia of hepatocytes and a higher incidence of microscopic hepatic venous invasion (9). The authors proposed that the peritumoral hyperintensity may be attributable to the regenerative changes of the peritumoral hepatocytes induced by tumor compression (9). We speculated that the degree of peri-tumoral hyperintensity reflects the degree of tumor compression of the surrounding hepatocytes, and might have some relationship with the biological behavior of hepatic NETs. The purpose of the present study was to identify the imaging and clinicopathological features of hepatic NETs associated with peritumoral hyperintensity in the HBP of gadoxetic acid-enhanced MR imaging.

\section{Patients and Methods}

Patients. Our institutional review board approved this study (IRB 201903807). The requirement for written informed consent was waived owing to the retrospective nature of the study. Between April 2014 and November 2018, 57 patients with pathologically proven hepatic NETs who underwent gadoxetic acid-enhanced MR imaging were enrolled. The pathological specimens were obtained from the hepatic lesion and/or the metastatic lesion. Of the 57 patients, 29 were men and 27 were women. The mean age of patients was 61.1 years (range $=28-85$ years). The primary sites of the metastatic lesions were small bowel $(n=30)$, pancreas $(n=11)$, lung $(n=10)$, rectum $(n=1)$, and unknown $(n=5)$. 
Table I. Comparison of the imaging findings among group 0, group 1, and group 2.

\begin{tabular}{|c|c|c|c|c|}
\hline & Group $0(\mathrm{n}=12)$ & Group $1(n=23)$ & Group $2(n=22)$ & $p$-Value \\
\hline Tumor size $(\mathrm{cm})$ & $4.0 \pm 2.3$ & $2.8 \pm 2.2$ & $2.7 \pm 1.5$ & N.S. \\
\hline T1WI & & & & N.S. \\
\hline Hypointense & $10(83.3 \%)$ & $18(78.3 \%)$ & $19(86.4 \%)$ & \\
\hline Isointense & $1(8.3 \%)$ & $5(21.7 \%)$ & $1(4.6 \%)$ & \\
\hline Hyperintense & $1(8.3 \%)$ & $0(0 \%)$ & $2(9.1 \%)$ & \\
\hline T2WI & & & & N.S. \\
\hline Hypointense & $1(8.3 \%)$ & $0(0 \%)$ & $0(0 \%)$ & \\
\hline Isointense & $0(0 \%)$ & $2(8.7 \%)$ & $3(13.6 \%)$ & \\
\hline Hyperintense & $11(91.7 \%)$ & $21(91.3 \%)$ & $19(86.4 \%)$ & \\
\hline ADC value $\left(\times 10^{-3} \mathrm{~mm}^{2} / \mathrm{s}\right)$ & $1.32 \pm 0.54$ & $0.83 \pm 0.02$ & $0.61 \pm 0.20$ & $<0.001$ \\
\hline Arterial enhancement pattern & & & & $<0.001$ \\
\hline Hypovascular & $5(41.7 \%)$ & $2(8.7 \%)$ & $1(4.6 \%)$ & \\
\hline Hypervascular & $4(33.3 \%)$ & $18(78.3 \%)$ & $5(22.7 \%)$ & \\
\hline Atypical & $3(25.0 \%)$ & $2(8.7 \%)$ & $16(72.7 \%)$ & \\
\hline
\end{tabular}

ADC: Apparent diffusion coefficient. Data presented as mean \pm standard deviation or as frequency (percentage).

MR techniques. Gadoxetic acid-enhanced MR imaging was performed using a clinical 1.5-T MR system (Avanto /Aera, Siemens, Erlangen, Germany) with a phased-array coil.

For the dynamic study, fat suppressed gradient-echo T1-weighted images with a three-dimensional (3D) acquisition sequence were obtained. For the gadoxetic acid-enhanced MRI, a multiphase dynamic study including arterial dominant, portal, late and hepatobiliary phases was performed. The total amount of gadoxetic acid (Eovist: Bayer Healthcare, Berlin, Germany) $(2.0 \mathrm{ml} / \mathrm{kg}$ body weight) was intravenously injected at the rate of $2.0 \mathrm{ml} / \mathrm{s}$, followed by a $25 \mathrm{ml}$ physiological saline flush using an automatic injector. We used the test injection method to determine the optimal scan timing of the arterial dominant phase. A test dose of $2 \mathrm{ml}$ of gadoxetic acid was injected and flushed with $25 \mathrm{ml}$ of physiological saline at the same injection rate. The detailed imaging parameters were as follows: quick fat saturation, relaxation time/echo time $(\mathrm{TR} / \mathrm{TE})=4.28 / 2.02 \mathrm{~ms}$; flip angle $(\mathrm{FA}) 10^{\circ}$; field of view (FOV) 42 $\mathrm{cm}$; matrix $192 \times 256$; slice thickness $=2.3 \mathrm{~mm}$.

Additionally, axial diffusion-weighed imaging (DWI), T1weighted imaging without fat suppression (FS), and T2-weighted imaging without FS were used in this study. The imaging parameters for axial DWI were as follows: spectral attenuated inversion recovery (SPAIR), TR/TE $=5,200 / 62 \mathrm{~ms}$; FOV $40 \mathrm{~cm}$; matrix 156×192; slice thickness $6 \mathrm{~mm}$; b-factors of 50, 400, and 800 $\mathrm{s} / \mathrm{mm}^{2}$. Apparent diffusion coefficient (ADC) maps were automatically generated on the operating console using all three images with b-factors of 50,400 , and $800 \mathrm{~s} / \mathrm{mm}^{2}$ and linear fitting. The imaging parameters for T1WI were: TR/TE=217/4.76 msec; FA $90^{\circ}$; FOV $40 \mathrm{~cm}$; matrix $192 \times 256$; slice thickness $5 \mathrm{~mm}$. The imaging parameters for T2WI were: TR/TE $=1100 / 182 \mathrm{msec}$; FA $150^{\circ}$; FOV $40 \mathrm{~cm}$; matrix $192 \times 256$; slice thickness $5 \mathrm{~mm}$.

Image analysis. Images of all axial sections of the lesions were independently evaluated by two radiologists (NF and $\mathrm{AH}$, with 15 and 5 years of experience in abdominal imaging, respectively). Both radiologists were blinded to the clinical and pathological results. In patients with multiple hepatic NETs, the largest lesion was evaluated. Lesions that exhibited apparent post-treatment change (e.g., after surgery, intervention, or ablation) were not evaluated. Peri-tumoral enhancement in the HBP was qualitatively defined as a hyperintense rim surrounding the tumor (9). Additionally, we divided the grade of peritumoral hyperintensity in the HBP according to the degree of the peritumoral hyperintense signal: group 0 (no peritumoral hyperintensity); group 1 (lower enhancement compared to the right/left hepatic duct or extrahepatic bile duct); and group 2 (equivalent to or greater enhancement compared to the right/left hepatic duct or extrahepatic bile duct). Interobserver agreement between the two radiologists was evaluated using weighted $\mathrm{K}$ statistics as follows: $\mathrm{K}$ value $<0.20$ : poor agreement; $0.20-0.39$ : fair agreement; 0.40-0.59: moderate agreement; 0.60-0.79: substantial agreement; and $>0.80$ : excellent agreement. Data analysis was performed after evaluating the results in a consensus manner.

Additionally, MR images were analyzed for the following parameters for each lesion: lesion maximal diameter; signal-intensity on T1-weighted and T2-weighted MR images (hypo-, iso-, or hyperintensity). The ADC value of the lesion was measured by placing a region of interest (ROI) on the solid portion of the lesion on the ADC map. Arterial enhancement pattern was classified into hypervascular, atypical (cholangiocarcinoma-like), or hypovascular pattern (10). Hypervascular pattern was defined as non-rim arterial phase hyperenhancement of the tumor. Atypical (cholangiocarcinoma-like) pattern was defined as rim enhancement in the arterial phase. Hypovascular pattern was defined as minimal or no enhancement during the arterial phase. These imaging features of each lesion were also evaluated by one radiologist $(\mathrm{NF}$, with 15 years of experience in abdominal imaging).

Progression-free survival rate of patients with hepatic NETs. We were able to obtain prognostic data pertaining to 43 of the 57 patients who were followed up at our institution. Progression-free survival rate was analyzed using computed tomography or MRI findings. Disease progression was defined as $\geq 20 \%$ progression in tumor size or the development of new lesions, according to the Response Criteria in Solid Tumors (RECIST) 1.1. The median follow-up period was 215 days (range $=51-1,175$ days). The progression-free survival rates were compared between the three groups. 
A

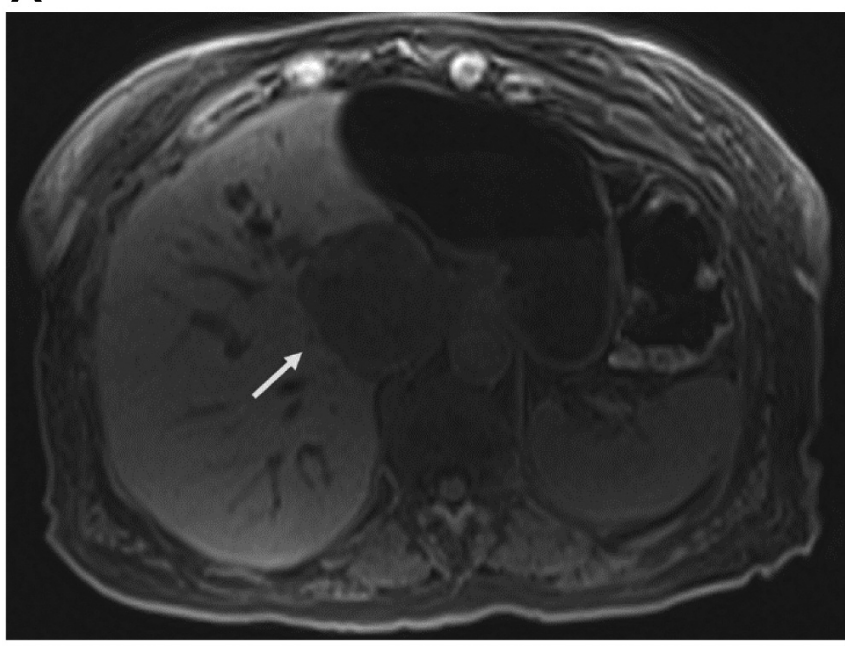

B

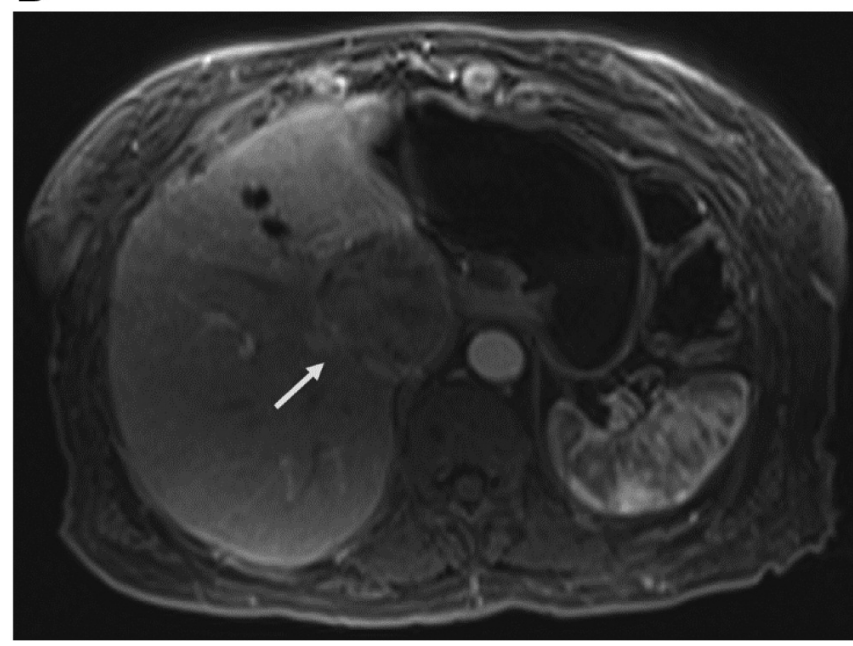

C

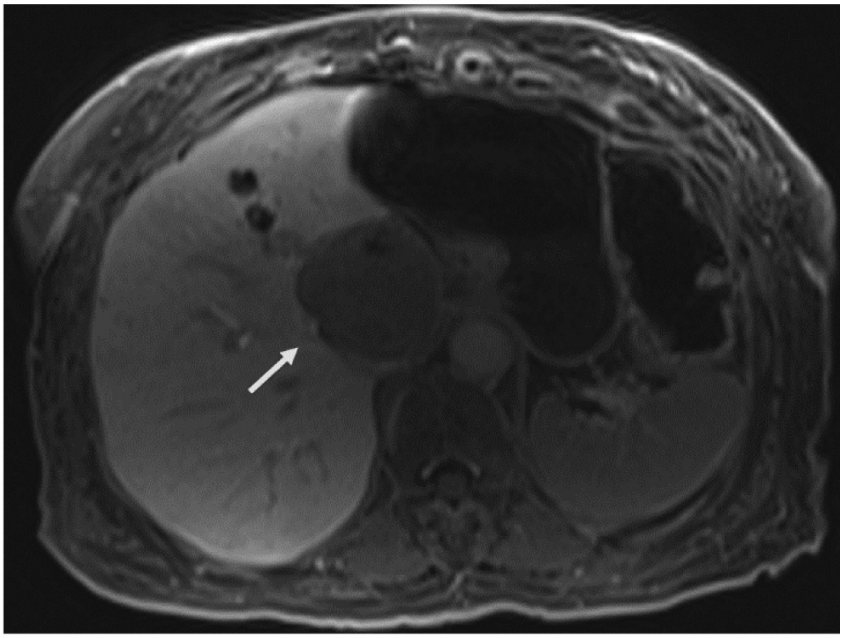

D

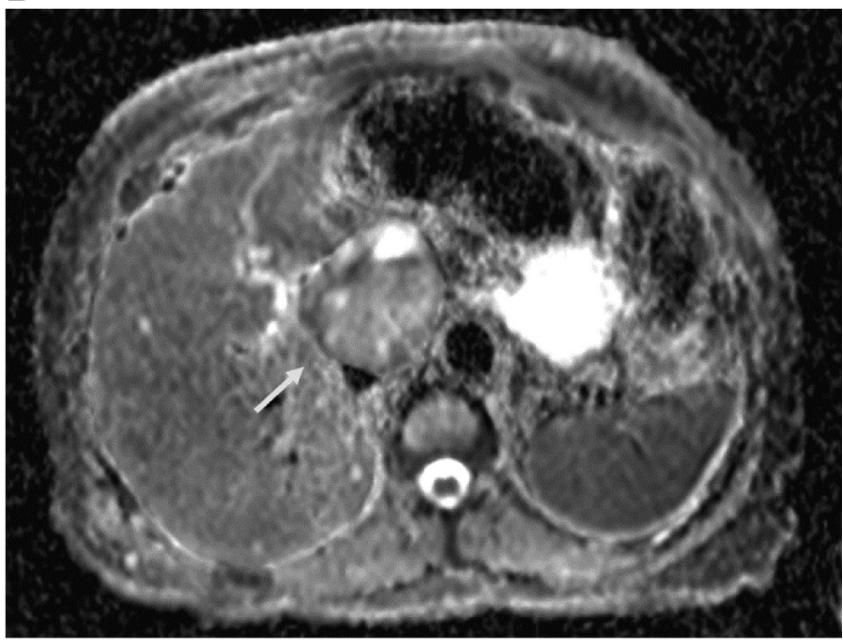

Figure 1. A 79-year-old woman with hepatic neuroendocrine tumor (arrow). On gadoxetic-enhanced MR imaging, the tumor shows hypointensity in the precontrast scan (A), appears hypovascular in the arterial phase $(B)$, and shows no peritumoral enhancement in the hepatobiliary phase $(C)$ (group 0). In the ADC map (D), the ADC value of the tumor was $1.52 \times 10^{-3} \mathrm{~mm}^{2} / \mathrm{s}$. ADC: Apparent diffusion coefficient.

Statistical analysis. The Kruskal-Wallis test and Mann-Whitney $U$ test were used to assess the correlation between the signal intensity pattern in the HBP and continuous variables (tumor size and ADC values). The Fisher's exact test was used to analyze the correlation between the signal intensity pattern in the HBP and nominal variables (arterial enhancement pattern, signal intensity on $\mathrm{T} 1$ weighed and T2-weighed images). Progression-free survival was evaluated using the Kaplan-Meier method and between-group differences assessed using the log-rank test. First, we compared these factors among the three lesion groups. Factors that showed a significant between-group difference were compared between each pair of groups. JMP 13.2.1 software (SAS Institute, Cary, NC, USA) was used for statistical analysis. $p$-Values $<0.05$ were considered indicative of statistical significance.

\section{Results}

Peritumoral intensity in the HBP and other imaging findings. Twelve patients $(21.1 \%)$ were classified as group 0,23 patients $(40.4 \%)$ as group 1 , and 22 patients $(38.6 \%)$ as group 2. There was excellent interobserver agreement in this respect (weighted K-value: 0.807 ).

The imaging findings in the three groups are summarized in Table I. The ADC values were significantly different among the three groups $\left(p=0.001\right.$, group $0,1.32 \pm 0.54 \times 10^{-3} \mathrm{~mm}^{2} / \mathrm{s}$; group $1,0.83 \pm 0.02 \times 10^{-3} \mathrm{~mm}^{2} / \mathrm{s}$; group $2,0.61 \pm 0.20 \times 10^{-3} \mathrm{~mm}^{2} / \mathrm{s}$ ). The ADC values of group 2 were significantly lower than those 
A

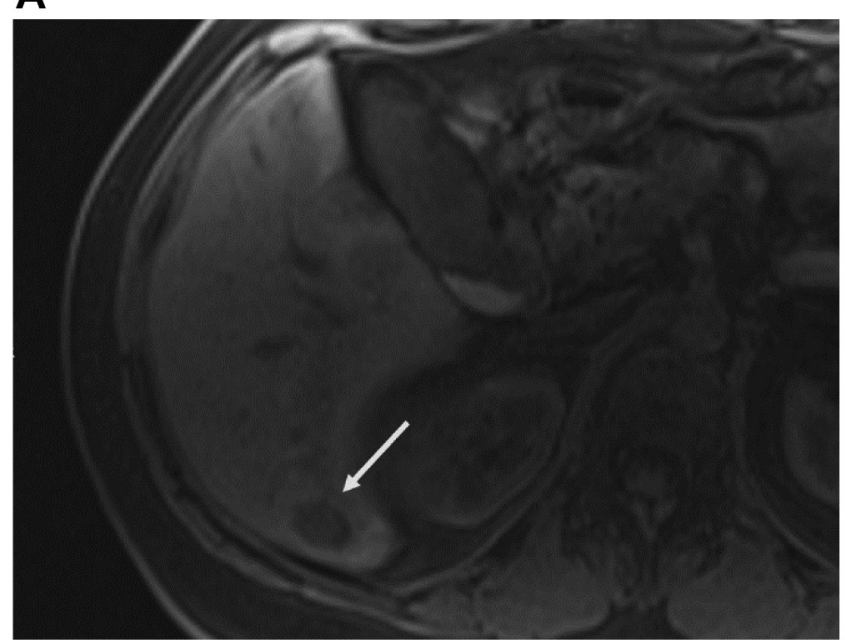

B

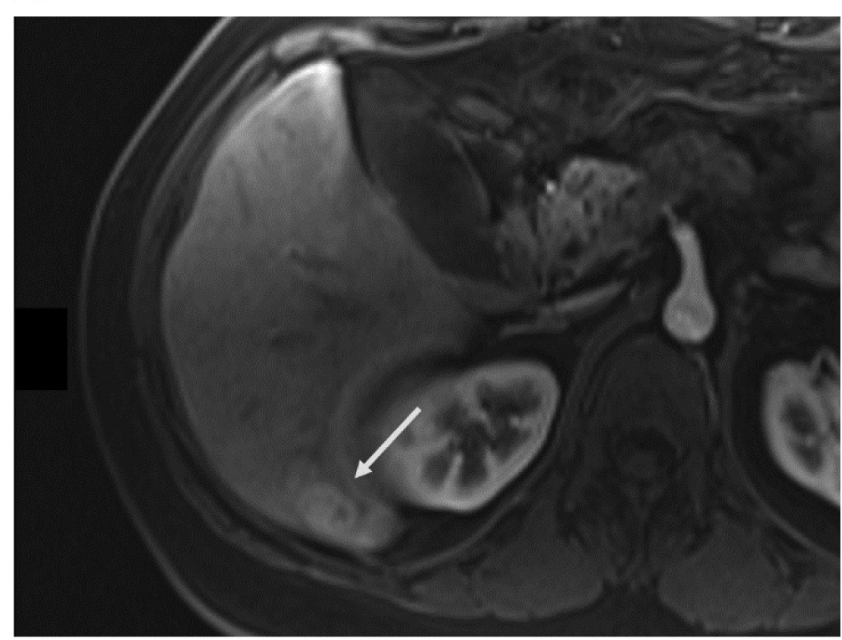

C

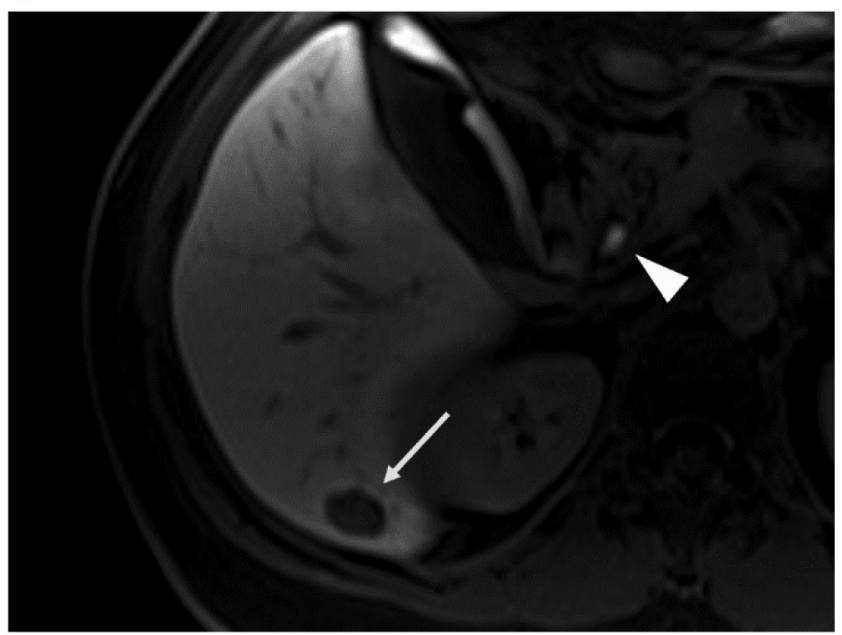

D

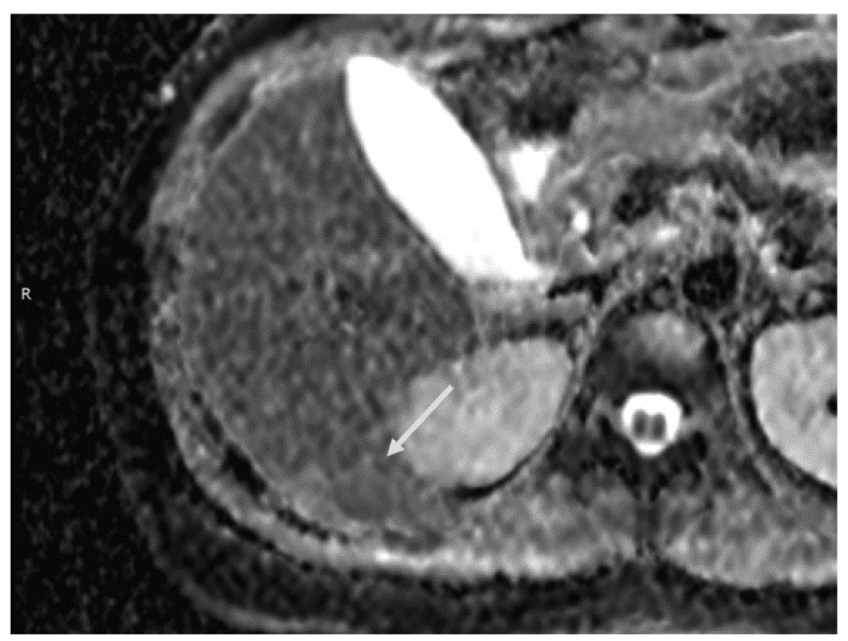

Figure 2. A 39-year-old woman with hepatic neuroendocrine tumor (arrow). On gadoxetic-enhanced MR imaging, the tumor shows hypointensity in the precontrast scan (A) and appears hypervascular in the arterial phase (B). In the hepatobiliary phase, the tumor shows mild peritumoral enhancement $(C)$, which is lower compared to the extrahepatic bile duct (arrowhead) (group 1). In the ADC map (D), the ADC value of the tumor was $0.88 \times 10^{-3} \mathrm{~mm}^{2} / \mathrm{s}$. ADC: Apparent diffusion coefficient.

of group $0(p<0.001)$ and group $1(p=0.002)$. The ADC values of group 1 were significantly lower than that of group 0 $(p=0.001)$.

The frequency of arterial enhancement pattern was significantly different among the three groups $(p<0.001)$. Atypical (cholangiocarcinoma-like) enhancement pattern in the arterial phase was significantly more frequently observed in group 2 tumors $(72.7 \%)$ as compared to that in group 0 $(25.0 \%)$ and group $1(8.7 \%)$ tumors $(p=0.012$ and $p<0.001$, respectively). Hypervascular pattern was significantly more frequently observed in group 1 tumors $(78.3 \%)$ as compared to that in group $0(33.3 \%)$ and group $2(22.7 \%)$ tumors $(p=0.024$ and $p<0.001$, respectively). Hypovascular pattern was significantly more frequently observed in group 0 tumors $(41.7 \%)$ than in group $2(4.6 \%)$ tumors $(p=0.014)$.

Tumor size and signal intensity on T1-weighted and T2weighted images were not significantly different among the three groups. Figure 1, Figure 2 and Figure 3 show the representative imaging findings for each of the lesion groups.

Peritumoral intensity in the $H B P$ and progression-free survival. Of 43 patients, 8 patients were classified as group 0,17 patients were classified as group 1 , and 18 patients were classified as group 2. Progression-free survival was significantly different among the three groups $(p=0.040)$ 
A

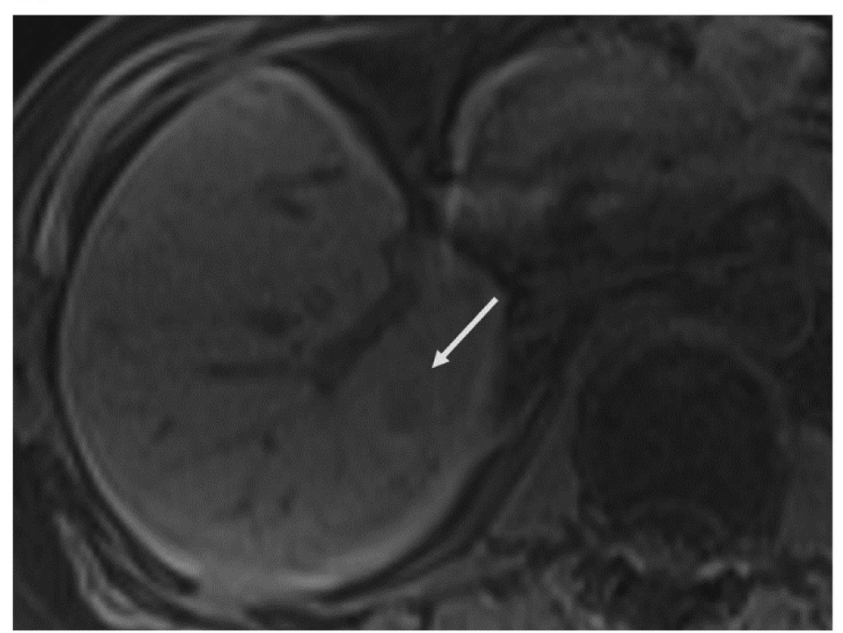

B

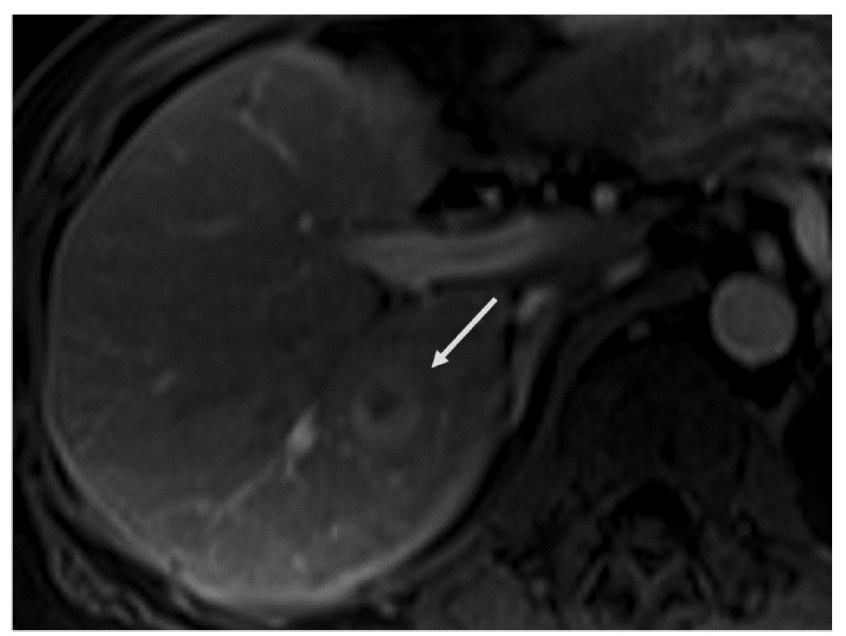

C

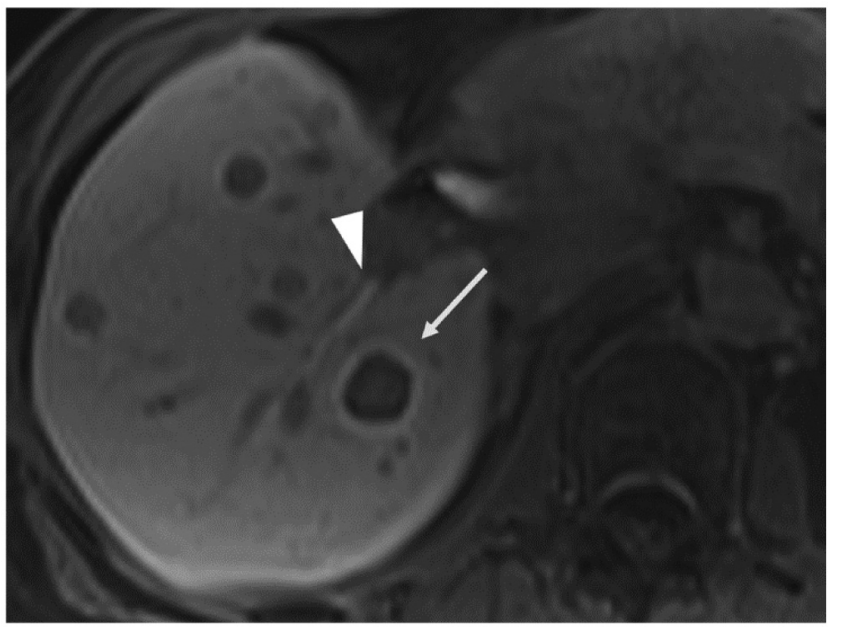

D

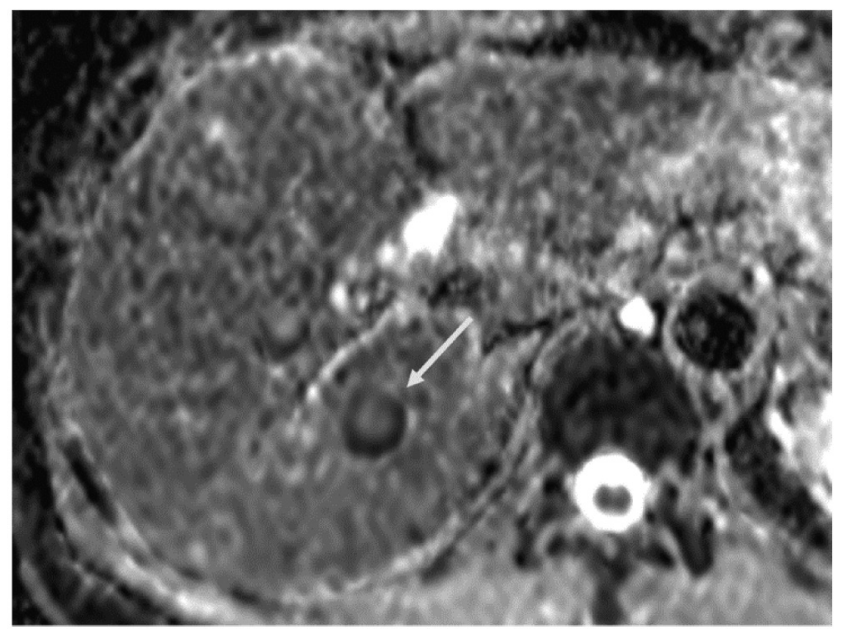

Figure 3. A 59-year-old woman with hepatic neuroendocrine tumor (arrow). On gadoxetic-enhanced magnetic resonance imaging, the tumor shows hypointensity in the precontrast scan (A) and has an atypical (cholangiocarcinoma-like) enhancement pattern in the arterial phase (B). In the hepatobiliary phase $(C)$, the tumor shows peritumoral enhancement in the hepatobiliary phase, which is equivalent to the right hepatic duct (arrowhead) (group 2). In the ADC map (D), the ADC value of the tumor was $0.58 \times 10^{-3} \mathrm{~mm}^{2} / \mathrm{s}$. ADC: Apparent diffusion coefficient.

(Figure 4). Patients in group 2 showed significantly poorer progression-free survival than patients in group $0(p=0.017)$. The disease-free survival rate was not significantly different between patients in group 0 and group 1, and between patients in group 1 and group 2 .

\section{Discussion}

Peritumoral enhancement in the HBP of gadoxetic acidenhanced MRI appears as a hyperintense rim surrounding the tumor $(9,11)$. Pathologically, peritumoral enhancement corresponds to peritumoral hyperplasia, defined as a rim of hyperplastic hepatocytes surrounding the tumor (9).
Peritumoral hyperplasia is observed in hepatic tumors, such as NETs, HCCs, and gastrointestinal stromal tumors (GISTs) $(9,11,12)$. There is no clear consensus on the pathogenesis of peritumoral hyperplasia. Regenerative changes of the hepatocytes induced by tumor compression is one of the potential causes of peritumoral hyperplasia (1). Tumors such as NETs, HCCs, and GISTs typically exhibit expansive growth unlike the infiltrative growth pattern of adenocarcinomas. The expansive growth can cause compression of the liver parenchyma and induce regenerative changes of hepatocytes, causing peritumoral enhancement.

In our study, hepatic NETs with greater degree of peritumoral enhancement were associated with lower ADC 


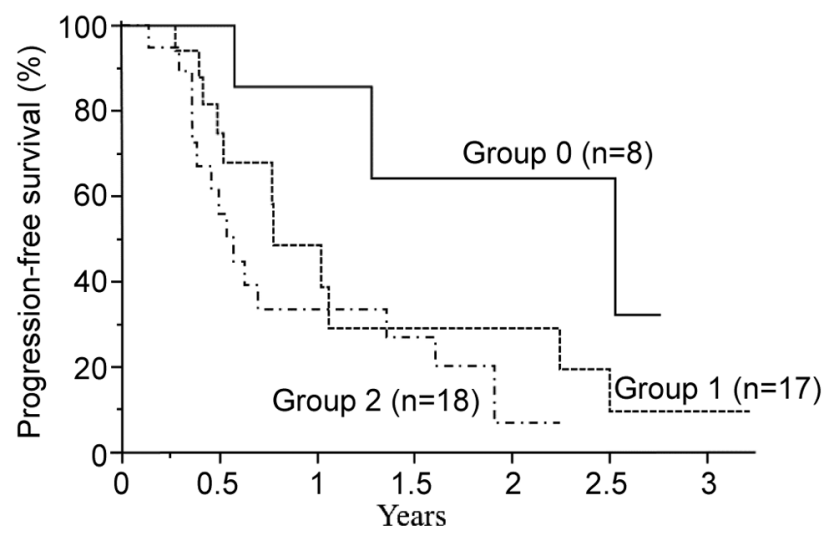

Figure 4. Progression-free survival curves of the patients in the three groups. The progression-free survival was significantly different among the three lesion groups $(p=0.040)$. Patients in group 2 showed significantly poorer progression-free survival than patients in group $0(p=0.017)$.

values. In addition, patients in group 2 showed significantly poorer progression-free survival than patients in group 0 . In previous studies, NETs with low ADC values showed an association with higher tumor grade and poorer prognosis $(10,13,14)$. Considering these results, in the present study, it can be said that hepatic NETs with greater peritumoral enhancement have greater malignant potential. It can be considered that NETs with greater malignant potential show more rapid expansive growth. We assume that the more rapid growth of NETs causes stronger compression of liver parenchyma, which in turn induces regenerative changes of hepatocytes and increases the degree of peritumoral enhancement. On the other hand, NETs with less malignant potential show less expansive growth and cause no or lower degree of peritumoral enhancement.

Additionally, in the present study, the frequency of arterial enhancement pattern was significantly different between the 3 lesion groups. Atypical (cholangiocarcinoma-like) enhancement pattern in the arterial phase was significantly more frequently observed in group 2 tumors. In a previous study, NETs that exhibited atypical (cholangiocarcinomalike) enhancement pattern had higher tumor grade (10). Therefore, this result supports our hypothesis that NETs with more malignant potential show greater degree of peritumoral enhancement. Hypervascular pattern, which is the most common pattern of hepatic NETs (10), was significantly more frequently observed in group 1 tumors. This result suggests that classic hepatic NETs commonly show peritumoral hyperintensity, which is typically hypointense compared to those of the bile duct. Hypovascular pattern was significantly more frequently observed in group 0 . In previous studies, hypovascular NETs showed an association with early tumor progression (15) or higher tumor grade
(16). However, in the present study, most of the patients (98.2\%) had received some treatment (such as surgery, intervention, bio-therapy, systemic chemotherapy, or ablation) at the time of MRI. Although we did not evaluate lesions that exhibited apparent post-treatment changes (such as surgery, intervention or ablation), we believe hypovascular NETs in the present study were caused by tumor necrosis or hemorrhage induced by treatment. We hypothesize that the treatment effect may have reduced the aggressiveness of the tumor or caused less expansive growth leading to the lack of peritumoral enhancement.

Other possible mechanisms of peritumoral hyperintensity have been considered: 1) perfusion abnormalities due to vascular invasion of the tumor $(9,12)$ and 2$)$ association of drainage flow (9). As for 1), considering the fact that the majority of the hepatic NETs are hematogeneous metastases, vascular invasion is common in hepatic NETs. For this reason, the presence of vascular invasion cannot explain our results. As for 2), we could evaluate drainage flow because the enhancement seen with gadoxetic acid was described as weak compared with that seen with the conventional extracellular contrast agents (17). However, Yoneda et al. reported that drainage flow from the tumor may not directly contribute to the formation of peritumoral hyperplasia in HCC (9). Therefore, we believe that the cause of peritumoral enhancement is mainly regenerative changes of the hepatocytes induced by tumor compression.

Some limitations of this study should be considered while interpreting our results. First, the evaluation of peritumoral hyperintensity was qualitative; quantitative evaluation was not performed. However, it is very difficult to accurately quantify peritumoral hyperintensity because of its small area. Additionally, the interobserver agreement between the two radiologists was excellent. Second, because patients with hepatic NETs are usually in advanced stage, most patients $(98.2 \%)$ had received some treatment during their long clinical course. We cannot deny the effect of such treatment prior to MRI on our results. Third, the study population did not consist of consecutive patients because gadoxetic acid-enhanced MR imaging was not performed in all patients with hepatic NETs. Therefore, selection bias may have led to an overestimation of our results. Fourth, we could not evaluate the pathological findings because liver biopsy or resection was performed only for a limited number of patients. Further studies are required to identify the relationship between imaging and pathological features including tumor grade.

In conclusion, hepatic NETs with greater peritumoral hyperintensity in the HBP of gadoxetic acid-enhanced MR imaging have more malignant potential. Evaluation of peritumoral hyperintensity in the HBP of gadoxetic acidenhanced MR imaging may help predict aggressive behavior of hepatic NETs. 


\section{Conflicts of Interest}

The Authors declare that they have no conflicts of interest in relation to this study.

\section{Authors' Contributions}

NF: Conceptualization, methodology, formal analysis, investigation, data curation, writing, project administration. AH: methodology, investigation, data curation. JP: Writing - review \& editing, supervision.

\section{References}

1 Kunz PL: Carcinoid and neuroendocrine tumors: building on success. J Clin Oncol 33(16): 1855-1863, 2015. PMID: 25918282. DOI: $10.1200 / J C O .2014 .60 .2532$

2 Ito T, Lee L and Jensen RT: Carcinoid-syndrome: recent advances, current status and controversies. Curr Opin Endocrinol Diabetes Obes 25(1): 22-35, 2018. PMID: 29120923. DOI: 10.1097/MED.0000000000000376

3 Riihimäki M, Hemminki A, Sundquist K, Sundquist J and Hemminki K: The epidemiology of metastases in neuroendocrine tumors. Int J Cancer 139(12): 2679-2686, 2016. PMID: 27553864 DOI: $10.1002 /$ ijc. 30400

4 Ito T, Igarashi H, Uehara H, Berna MJ and Jensen RT: Causes of death and prognostic factors in multiple endocrine neoplasia type 1: a prospective study: comparison of $106 \mathrm{MEN} 1 /$ ZollingerEllison syndrome patients with 1613 literature MEN1 patients with or without pancreatic endocrine tumors. Medicine (Baltimore) 92(3): 135-181, 2013. PMID: 23645327. DOI: 10.1097/MD.0b013e3182954af1

5 Vogl TJ, Kümmel S, Hammerstingl R, Schellenbeck M, Schumacher G, Balzer T, Schwarz W, Müller PK, Bechstein WO, Mack MG, Söllner $O$ and Felix R: Liver tumors: comparison of MR imaging with Gd-EOB-DTPA and Gd-DTPA. Radiology 200(1): 59-67, 1996. PMID: 8657946. DOI: 10.1148/ radiology.200.1.8657946

6 Huppertz A, Balzer T, Blakeborough A, Breuer J, Giovagnoni A, Heinz-Peer G, Laniado M, Manfredi RM, Mathieu DG, Mueller D, Reimer P, Robinson PJ, Strotzer M, Taupitz M, Vogl TJ and European EOB Study Group: Improved detection of focal liver lesions at MR imaging: multicenter comparison of gadoxetic acid-enhanced MR images with intraoperative findings. Radiology 230(1): 266-275, 2004. PMID: 14695400. DOI: 10.1148/radiol.2301020269

7 Motosugi U, Ichikawa T, Morisaka H, Sou H, Muhi A, Kimura $\mathrm{K}$, Sano K and Araki T: Detection of pancreatic carcinoma and liver metastases with gadoxetic acid-enhanced MR imaging: comparison with contrast-enhanced multi-detector row CT Radiology 260(2): 446-453, 2011. PMID: 21693662. DOI: 10.1148/radiol.11103548

8 Muhi A, Ichikawa T, Motosugi U, Sou H, Nakajima H, Sano K, Sano M, Kato S, Kitamura T, Fatima Z, Fukushima K, Iino H, Mori Y, Fujii H and Araki T: Diagnosis of colorectal hepatic metastases: comparison of contrast-enhanced CT, contrastenhanced US, superparamagnetic iron oxide-enhanced MRI, and gadoxetic acid-enhanced MRI. J Magn Reson Imaging 34(2): 326-335, 2011. PMID: 21780227. DOI: 10.1002/jmri.22613
9 Yoneda N, Matsui O, Kitao A, Komori T, Kozaka K, Ikeda H, Yoshida K, Inoue D, Minami T, Koda W, Kobayashi S and Gabata T: Peri-tumoral hyperintensity on hepatobiliary phase of gadoxetic acid-enhanced MRI in hepatocellular carcinomas: correlation with peri-tumoral hyperplasia and its pathological features. Abdom Radiol (NY) 43(8): 2103-2112, 2018. PMID: 29260280. DOI: $10.1007 / \mathrm{s} 00261-017-1437-4$

10 Min JH, Kang TW, Kim YK, Kim SH, Shin KS, Lee JE, Ha SY and Sohn I: Hepatic neuroendocrine tumour: Apparent diffusion coefficient as a potential marker of prognosis associated with tumour grade and overall survival. Eur Radiol 28(6): 2561-2571, 2018. PMID: 29368162. DOI: 10.1007/s00330-017-5248-3

11 Fujita N, Nishie A, Asayama Y, Ishigami K, Ushijima Y, Kakihara D, Nakayama T, Morita K, Ishimatsu K and Honda H: Hyperintense liver masses at hepatobiliary phase gadoxetic acidenhanced MRI: Imaging appearances and clinical importance. Radiographics 40(1): 72-94, 2020. PMID: 31834849. DOI: 10.1148/rg.2020190037

12 Arnason T, Fleming KE and Wanless IR: Peritumoral hyperplasia of the liver: a response to portal vein invasion by hypervascular neoplasms. Histopathology 62(3): 458-464, 2013. PMID: 23240735. DOI: 10.1111/his.12032

13 Kim M, Kang TW, Kim YK, Kim SH, Kwon W, Ha SY and Ji SA: Pancreatic neuroendocrine tumour: Correlation of apparent diffusion coefficient or WHO classification with recurrence-free survival. Eur J Radiol 85(3): 680-687, 2016. PMID: 26826888. DOI: $10.1016 /$ j.ejrad.2015.12.029

14 Lotfalizadeh E, Ronot M, Wagner M, Cros J, Couvelard A, Vullierme MP, Allaham W, Hentic O, Ruzniewski P and Vilgrain V: Prediction of pancreatic neuroendocrine tumour grade with MR imaging features: added value of diffusion-weighted imaging. Eur Radiol 27(4): 1748-1759, 2017. PMID: 27543074. DOI: $10.1007 /$ s00330-016-4539-4

15 Denecke T, Baur AD, Ihm C, Steffen IG, Tischer E, Arsenic R, Pascher A, Wiedenmann B and Pavel M: Evaluation of radiological prognostic factors of hepatic metastases in patients with non-functional pancreatic neuroendocrine tumors. Eur J Radiol 82(10): e550-e555, 2013. PMID: 23891296. DOI: 10.1016/j.ejrad.2013.06.017

$16 \mathrm{Kim}$ JH, Eun HW, Kim YJ, Han JK and Choi BI: Staging accuracy of MR for pancreatic neuroendocrine tumor and imaging findings according to the tumor grade. Abdom Imaging 38(5): 1106-1114, 2013. PMID: 23728305. DOI: 10.1007/ s00261-013-0011-y

17 Santillan C, Fowler K, Kono Y and Chernyak V: LI-RADS major features: CT, MRI with extracellular agents, and MRI with hepatobiliary agents. Abdom Radiol (NY) 43(1): 75-81, 2018. PMID: 28828680. DOI: 10.1007/s00261-017-1291-4
Received November 17, 2021

Revised December 5, 2021

Accepted December 6, 2021 